\title{
Sudden Disappearance of the Blood Flow in a Case of Pancreatic Acinar Cell Carcinoma
}

\author{
Atsushi Kanno ${ }^{1}$, Atsushi Masamune ${ }^{1}$, Shin Hamada ${ }^{1}$, Kazuhiro Kikuta ${ }^{1}$, Kiyoshi Kume ${ }^{1}$, \\ Morihisa Hirota ${ }^{1}$, Kentaro Shima ${ }^{2}$, Takaho Okada ${ }^{2}$, Fuyuhiko Motoi ${ }^{2}$, Fumiyoshi Fujishima ${ }^{3}$, \\ Kazuyuki Ishida ${ }^{4}$, Michiaki Unno ${ }^{2}$ and Tooru Shimosegawa ${ }^{1}$
}

\begin{abstract}
A 55-year-old man was referred to our hospital for a further examination of a pancreatic cystic tumor with a solid component exhibiting vascularity. A few days later, the patient was admitted with a complaint of sudden severe epigastric pain. Enhanced CT showed the loss of vascularity in the tumor. In particular, contrastenhanced endoscopic ultrasonography (EUS) clearly demonstrated the disappearance of the blood flow, and a histological examination revealed acinar cell carcinoma with central necrosis. To our knowledge, this is the first case in the literature of acinar cell carcinoma associated with the sudden disappearance of vascularity. In this case, contrast-enhanced harmonic EUS was especially useful for assessing the degree of vascularity.
\end{abstract}

Key words: computed tomography, contrast-enhanced harmonic EUS, pancreatoduodenectomy, pancreatic tumor

(Intern Med 53: 2589-2593, 2014)

(DOI: 10.2169/internalmedicine.53.2859)

\section{Introduction}

Acinar cell carcinoma (ACC) is a very rare type of pancreatic exocrine tumor, accounting for less than $1 \%$ of all pancreatic tumors. The definitive diagnosis of ACC requires a histopathological analysis (1), and ACCs are generally well-circumscribed, solid, cellular, medullary neoplasms, although some lesions exhibit the characteristics of intraductal growth (2) and cystic formation. With respect to differentiating this tumor from other pancreatic masses, such as neuroendocrine tumors, pancreatoblastoma and solid pseudopapillary neoplasms, electron microscopy is sometimes required to identify zymogen granules. To date, only a few studies have reported the imaging appearance of $\operatorname{ACC}(3,4)$. Since the radiologic and blood flow patterns of ACC are generally nonspecific, it is often difficult to diagnose the condition based on only imaging studies.

Endoscopic ultrasonography (EUS) has the advantage of being able to obtain high-resolution images of the pancreas, which is surrounded by the small intestine and duodenum (5). However, the utility of EUS remains limited to assessing vascularity in the pancreas. In contrast, contrastenhanced harmonic EUS (CH-EUS) is a novel technology for visualizing sites of vascularity in detail (6) and has been reported to be particularly useful for assessing the degree of vascularity in pancreatic tumors $(7,8)$. We herein report a case of ACC associated with the sudden disappearance of the blood flow in the tumor, in which CH-EUS was useful for assessing changes in vascularity.

\section{Case Report}

A 53-year-old man with a past history of hypertension was admitted to another hospital due to epigastric pain in October 2010. A physical exam showed moderate epigastric tenderness but no active signs of peritoneal irritation. The initial laboratory data revealed slight elevation of the lipase

\footnotetext{
${ }^{1}$ Division of Gastroenterology, Tohoku University Graduate School of Medicine, Japan, ${ }^{2}$ Division of Hepato-biliary-Pancreatic Surgery, Tohoku University Graduate School of Medicine, Japan, ${ }^{3}$ Department of Pathology, Tohoku University Graduate School of Medicine, Japan and ${ }^{4}$ Department of Diagnostic Pathology, Iwate Medical University School of Medicine, Japan Received for publication March 11, 2014; Accepted for publication June 22, 2014
} Correspondence to Dr. Atsushi Kanno, atsushih@med.tohoku.ac.jp 

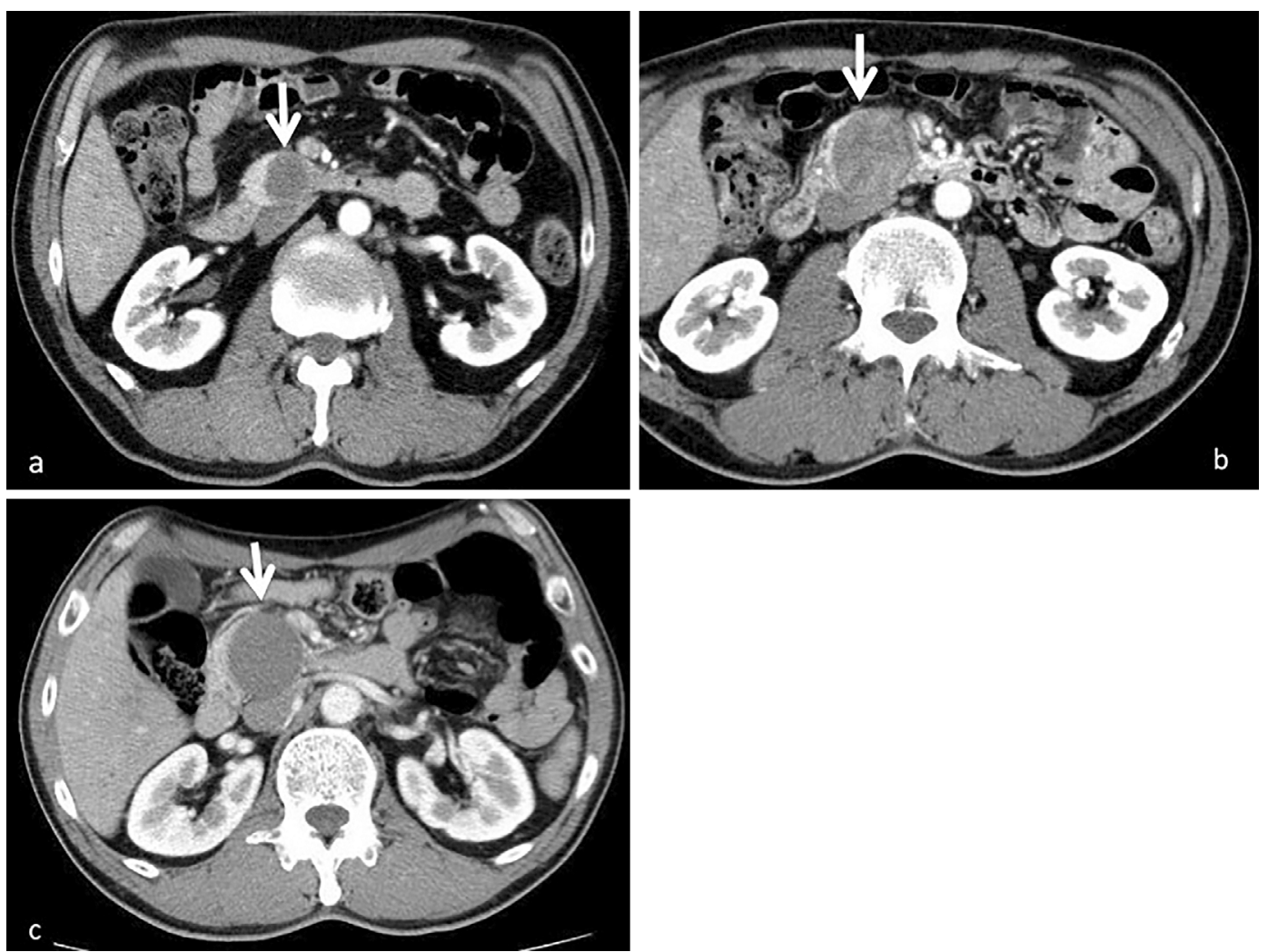

Figure 1. Enhanced CT findings. a: CT performed in November 2010 revealed a small cystic lesion in the pancreatic head (arrow). b: CT performed in November 2011 showed that the cystic tumor had grown larger in size and contained medullary areas (arrow). c: CT performed in December 2011 after the sudden onset of epigastric pain demonstrated the disappearance of vascularity in the solid component (arrow).

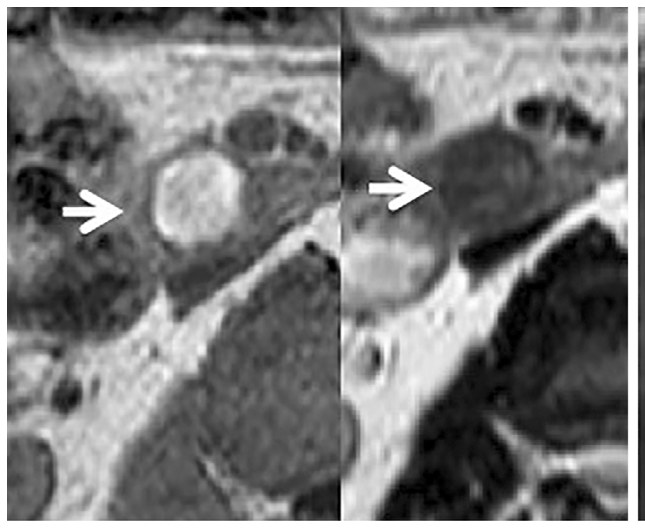

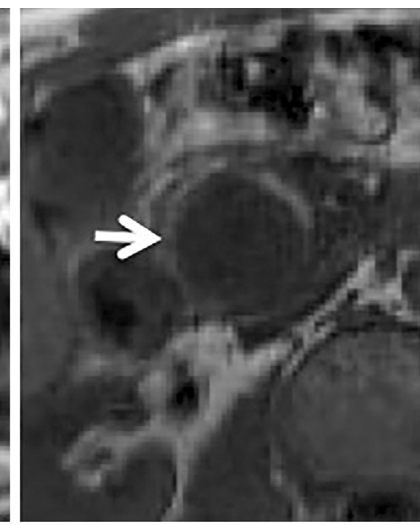

C

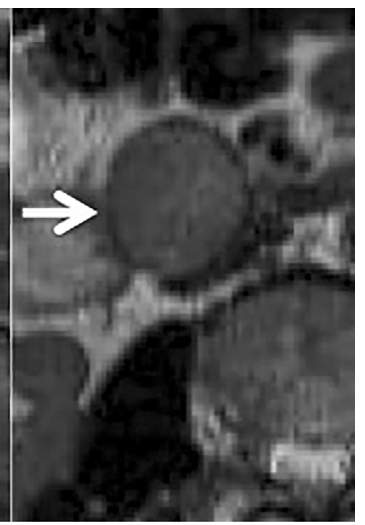

d

Figure 2. Intensity of MRI changes in the tumor. a: T1-weighted image obtained in November 2010. b: T2-weighted image obtained in November 2010. c: T1-weighted image obtained in November 2011. d: T2-weighted image obtained in November 2011. The intensity on T1/T2-weighted images of MRI changed from high/low to low/slightly high, respectively (arrows).

level (serum lipase: $62 \mathrm{IU} / \mathrm{L}$ ); however, the blood cell count and serum amylase and C-reactive protein (CRP) levels were within the normal ranges. In addition, tumor markers, including carcinoembryonic antigen (CEA) and CA19-9, were not elevated, and computed tomography (CT) did not reveal any significant findings of pancreatitis; however, a small cystic lesion was in the pancreatic head. Although the pa- tient's symptoms improved spontaneously, he was referred to our hospital for a further examination of the pancreatic cystic lesion in November 2010. At that time, CT revealed a small cystic lesion in the pancreatic head (Fig. 1a), and magnetic resonance imaging (MRI) showed a high- and lowintensity lesion on T1- and T2-weighted images (Fig. 2a, b), respectively. We suspected that the cystic lesion was accom- 


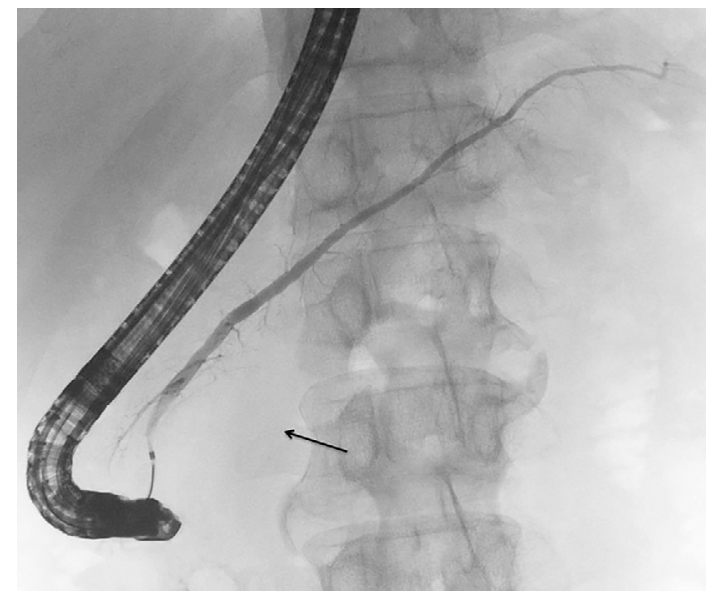

Figure 3. ERCP revealed no connection between the cystic lesion and pancreatic duct. ERCP showed some of the pancreatic branches to be compressed by the tumor (arrow).

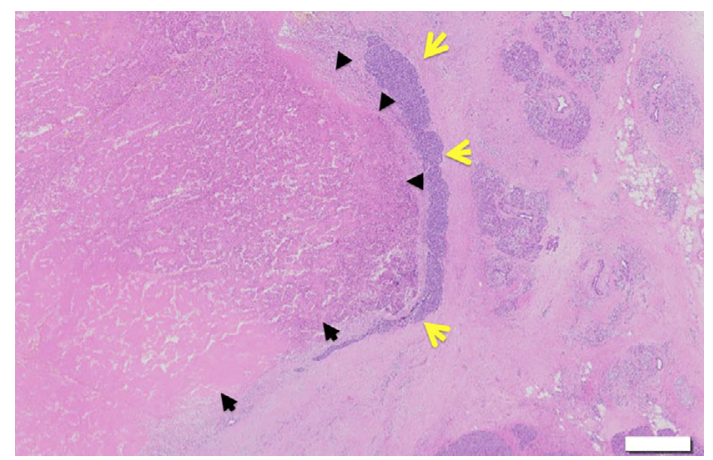

Figure 5. The histopathological findings revealed acinar cell carcinomas (arrows) along the periphery of necrotic tissue (surrounded by arrowheads).

panied by hemorrhagic changes. However, endoscopic retrograde cholangiopancreatography (ERCP) findings did not demonstrate a connection between the pancreatic cyst and the pancreatic duct (Fig. 3), and we therefore decided to follow the patient based on a diagnosis of a simple pancreatic cyst with hemorrhage. In November 2011, CT showed growth of the cystic lesion, and enhanced CT revealed a medullary tumor with weak vascularity within the cyst (Fig. 1b). Furthermore, the MRI findings changed from low to slightly high intensity on T1- and T2-weighted images (Fig. 2c, d), respectively. Meanwhile, EUS demonstrated a solid component in the pancreatic cystic lesion, and $\mathrm{CH}-$ EUS performed with contrast agent (Sonazoid $^{\circledR}$, Daiichi Sankyo Pharmaceutical, Tokyo, Japan) detected vascularity in the solid tumor within the cyst (Fig. 4a). While waiting to examine the patient further, he was admitted with sudden severe epigastric pain in December 2011. Upon admission, the laboratory data showed slight elevation of the CRP (1.5 $\mathrm{mg} / \mathrm{dL}$ ) and serum lipase (54 U/L) levels; however, the serum amylase level and blood cell count were within the normal ranges, as were tumor markers, including CEA, CA19-9 and $\alpha$-fetoprotein (AFP). A repeat CT examination revealed the disappearance of vascularity in the tumor, with no find-

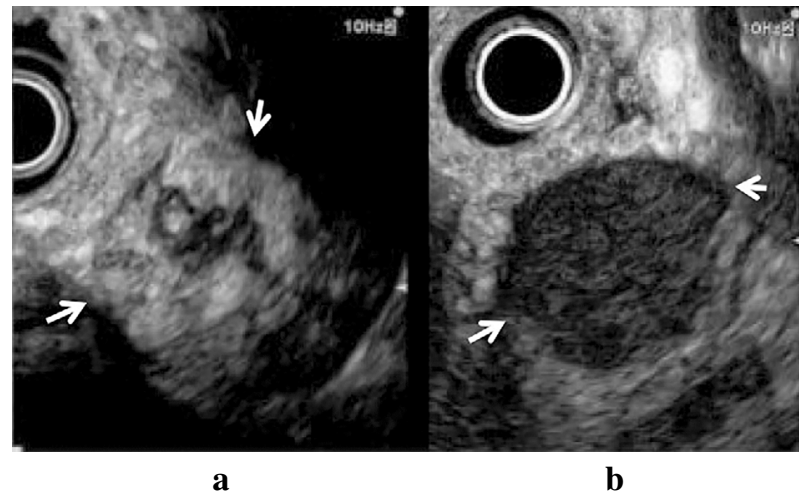

Figure 4. Contrast-enhanced harmonic EUS (CEH-EUS) revealed the disappearance of vascularity in the tumor more clearly. (a) CEH-EUS performed in November 2011 revealed hypervascularity in the tumor (arrows). (b) CEH-EUS showed that the blood flow in the tumor had vanished completely (arrows).

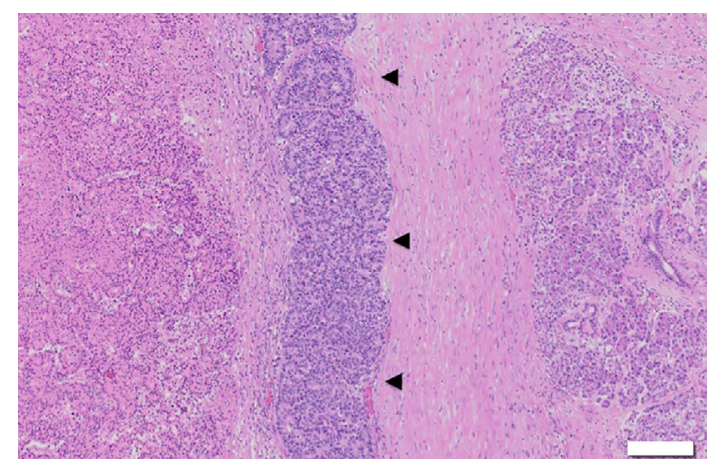

Figure 6. The microscopic findings showed an acinar pattern with neoplastic cells (arrowheads).

ings of pancreatitis (Fig. 1c). Although EUS did not identify any changes in the tumor, CH-EUS showed the disappearance of the blood flow in the tumor more clearly than enhanced CT (Fig. 4b). The patient's symptoms subsequently improved spontaneously, and we diagnosed the cystic lesion as a viable malignant tumor with the sudden onset of necrosis, such as a neuroendocrine tumor, acinar cell carcinoma or solid pseudopapillary neoplasm. Based on this diagnosis, pancreatoduodenectomy reconstructed according to Child's procedure with resection of the regional lymph nodes was performed in January 2012.

The tumor, which measured $35 \times 30 \mathrm{~mm}$, was located in the head of the pancreas. The gross features of the resected pancreatic specimens revealed a circumscribed encapsulated tumor; however, no invasion to the duodenum was evident. The cut surface was white and solid, without cystic changes. Microscopically, most of the tumor comprised necrotic tissue; however, some small portions around the tumor contained small round cells with amphophilic fine granular cytoplasm (Fig. 5, 6). No thrombi were detected in the feeding artery of the tumor. Immunohistochemically, the tumor cells were positive for $\alpha-1$ antichymotrypsin, CK7 and CK19 and negative for chromogranin $\mathrm{A}$ and synaptophysin (Fig. 7); 

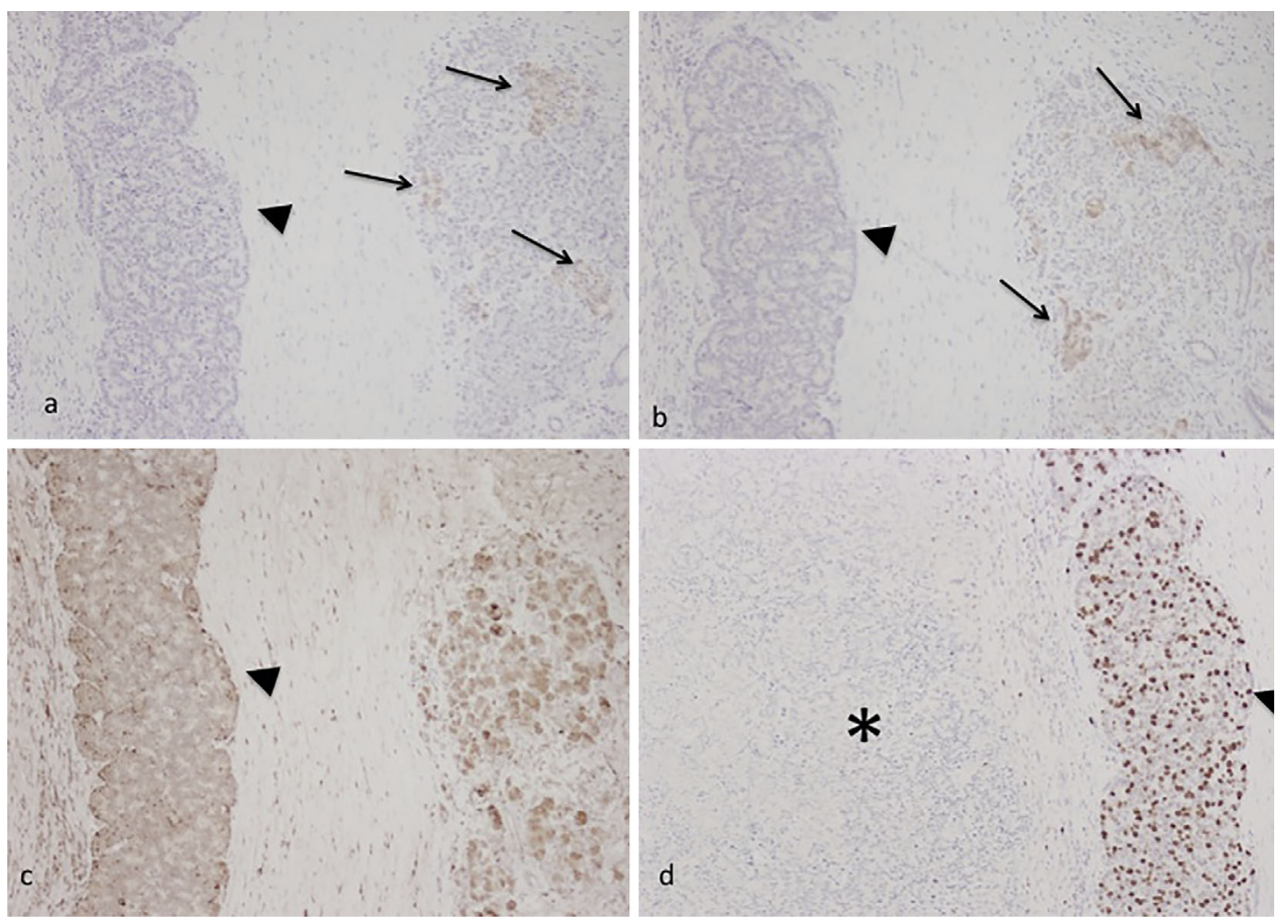

Figure 7. Immune staining. (a) Chromogranin was negative in the tumor (arrowhead); however, normal islet cells were positive (arrows). (b) Synaptophysin was negative in the tumor (arrowhead); however, normal islet cells were positive (arrows). (c) Alpha-antichymotrypsin was positive. (d) The Ki-67 index of the tumor was $40 \%$; however, the necrotic portion was negative (*).

these findings were consistent with a diagnosis of ACC. The histopathological diagnosis was therefore ACC with necrosis. There were no postoperative complications, and the patient was treated with adjuvant chemotherapy using gemcitabine and S-1 for six months according to the current regimen for adjuvant chemotherapy for pancreatic cancer used in our hospital. The patient has since survived for 24 months without recurrence.

\section{Discussion}

$\mathrm{ACC}$ is a rare neoplasm that arises from acinar elements of the exocrine pancreas, accounting for approximately $1 \%$ of all pancreatic neoplasms. Since there are few case reports and case series of ACC, its clinical and imaging features, treatment and outcomes have not been fully investigated (9-11). Hsu et al. summarized the radiological findings of six cases of ACC (3). In that series, most the lesions were distributed in the pancreatic head, with a tumor size ranging from 3.5 to $12 \mathrm{~cm}$. The tumor margins were classified as uniformly well defined, partially obscured or ill defined, and five of the six cases involved central hypodensity and/or necrosis. MRI showed several intensity patterns based on the contents of the tumor, including hemorrhage, necrosis, cystic degeneration and calcification. Since there are limited data regarding the imaging features of ACC, it is difficult to diagnose this tumor correctly using imaging studies. The vascularity of ACC has also not been well de- scribed, although examinations of both dynamic CT and MRI images have revealed that the tumors exhibit heterogeneous enhancement, albeit less than that observed in the surrounding normal parenchyma. In some cases of ACC, the blood flow disappears due to necrosis; however, there are no compatible patterns of vascularity of ACC. We herein report the case of a patient with ACC whose tumor demonstrated a sudden disappearance in blood flow. To our knowledge, this is the first report of ACC with the sudden disappearance of vascularity in the literature. Although we speculate that thrombi developed in the feeding artery of the tumor in this case, a histological examination did not reveal the cause of the sudden disappearance in blood flow. Mohammadi et al. reported a case of ACC with spontaneous rupture (12). Histological studies of ACC have also revealed frequent necrotic changes (1). Furthermore, histological examinations of ACCs have demonstrated half of such lesions to show vascular invasion (1) and/or hemorrhagic or necrotic changes (11). These reports suggest that ACC is associated with vascular events.

Although enhanced CT revealed a disappearance in blood flow in the present case, CH-EUS demonstrated the disappearance in vascularity more clearly. Regarding the diagnosis of pancreatic tumors, EUS is superior to other available imaging modalities due to its excellent resolution (6). However, vascular images cannot be obtained using only B-mode EUS. Although CT can be used to acquire enhanced images at a specific time, it is not useful for detecting changes in 
weak vascularity, as observed in the current tumor. On the other hand, $\mathrm{CH}$-EUS makes it possible to assess the degree of vascularity in some lesions $(7,8)$. CH-EUS is also expected to be useful for diagnosing various pancreatic tumors based on the detection of an enhancing pattern, as this modality can be used to obtain sequential enhanced images. Furthermore, CH-EUS is superior to enhanced CT because it does not require exposure to radiation.

It is difficult to diagnose ACC correctly prior to surgery, as some tumors mimic ACC both cytologically and histologically, including neuroendocrine tumors, solid pseudopapillary neoplasms and pancreatic ductal adenocarcinomas with medullary growth (13). The gold standard for diagnosing solid pancreatic lesions is a pathological examination of histological specimens obtained via surgery or EUSguided fine-needle aspiration (EUS-FNA). However, we did not perform EUS-FNA to obtain a histological diagnosis in this case because other imaging modalities showed a disappearance in the blood flow of the lesion. Indeed, since the histological findings showed that most of the tumor contained necrotic tissue, we may not have been able to diagnose the mass as an ACC based on the histological findings of EUS-FNA. Generally, ACC can be difficult to diagnose histologically due to the presence of necrosis.

Since it is difficult to diagnose ACC correctly prior to surgery, the diagnosis must be made based on several factors. For example, elevated lipase has been identified in half of ACC patients. Several studies have also reported elevated serum AFP levels in patients with ACC (13). However, the rate of positivity for these factors is not high. In fact, the serum AFP level was not elevated in our patient. Due to the rarity of ACC, it is necessary to coordinate multicenter studies in order to clarify the clinical aspects of and establish a method of treatment for this lesion.

In conclusion, we experienced a case of ACC associated with the sudden disappearance of the blood flow within the tumor. This clinical course of ACC has not been previously reported. Therefore, physicians should recognize that several clinical symptoms can occur in patients with ACC, particu- larly vascular events. CH-EUS can be used to identify such complications more clearly.

The authors state that they have no Conflict of Interest (COI).

\section{References}

1. La Rosa S, Adsay V, Albarello L, et al. Clinicopathologic study of 62 acinar cell carcinomas of the pancreas: insights into the morphology and immunophenotype and search for prognostic markers. Am J Surg Pathol 36: 1782-1795, 2012.

2. Kim HJ, Kim YK, Jang KT, Lim JH. Intraductal growing acinar cell carcinoma of the pancreas. Abdom Imaging 38: 1115-1119, 2013.

3. Hsu MY, Pan KT, Chu SY, Hung CF, Wu RC, Tseng JH. CT and MRI features of acinar cell carcinoma of the pancreas with pathological correlations. Clin Radiol 65: 223-229, 2010.

4. Hu S, Hu S, Wang M, Wu Z, Miao F. Clinical and CT imaging features of pancreatic acinar cell carcinoma. Radiol Med 118: 723-731, 2013.

5. Fusaroli P, Kypraios D, Caletti G, Eloubeidi MA. Pancreaticobiliary endoscopic ultrasound: a systematic review of the levels of evidence, performance and outcomes. World J Gastroenterol 18: 4243-4256, 2012.

6. Hirooka Y, Itoh A, Kawashima H, et al. Contrast-enhanced endoscopic ultrasonography in digestive diseases. J Gastroenterol 47: 1063-1072, 2012.

7. Kitano M, Sakamoto H, Kudo M. Endoscopic ultrasound: contrast enhancement. Gastrointest Endosc Clin N Am 22: 349-358, 2012.

8. Kitano M, Sakamoto H, Kudo M. Contrast-enhanced endoscopic ultrasound. Dig Endosc 26 (Suppl 1): 79-85, 2014.

9. Chen J, Baithun SI. Morphological study of 391 cases of exocrine pancreatic tumours with special reference to the classification of exocrine pancreatic carcinoma. J Pathol 146: 17-29, 1985.

10. Seth AK, Argani P, Campbell KA, et al. Acinar cell carcinoma of the pancreas: an institutional series of resected patients and review of the current literature. J Gastrointest Surg 12: 1061-1067, 2008.

11. Ordonez NG. Pancreatic acinar cell carcinoma. Adv Anat Pathol 8: 144-159, 2001.

12. Mohammadi A, Porghasem J, Esmaeili A, Ghasemi-Rad M. Spontaneous rupture of a pancreatic acinar cell carcinoma presenting as an acute abdomen. Int J Surg Case Rep 3: 293-295, 2012.

13. Toll AD, Hruban RH, Ali SZ. Acinar cell carcinoma of the pancreas: clinical and cytomorphologic characteristics. Korean J Pathol 47: 93-99, 2013.

(C) 2014 The Japanese Society of Internal Medicine http://www.naika.or.jp/imonline/index.html 